\title{
A simple method to measure the temperature and levitation height of devices rotating at cryogenic temperatures
}

Cite as: Rev. Sci. Instrum. 91, 045118 (2020); https://doi.org/10.1063/5.0005498

Submitted: 21 February 2020 . Accepted: 06 April 2020 . Published Online: 27 April 2020

Paolo de Bernardis (D), Fabio Columbro (D), Silvia Masi (D), Alessandro Paiella (D), and Giovanni Romeo (i)

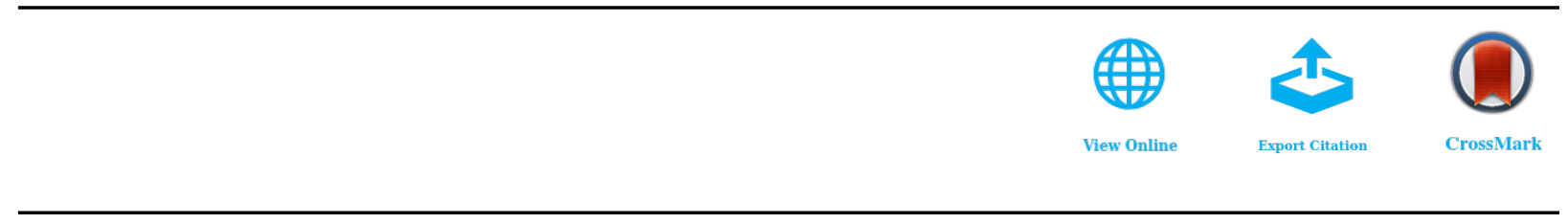

\section{ARTICLES YOU MAY BE INTERESTED IN}

A levitated magnetic dipole configuration as a compact charged particle trap

Review of Scientific Instruments 91, 043507 (2020); https://doi.org/10.1063/1.5142863

Fast switching coil system for sample premagnetization in an unshielded ultra-low-field nuclear magnetic resonance experiment

Review of Scientific Instruments 91, 045119 (2020); https://doi.org/10.1063/5.0003882

An innovative experimental device for borehole ballooning

Review of Scientific Instruments 91, 046103 (2020); https://doi.org/10.1063/1.5139950

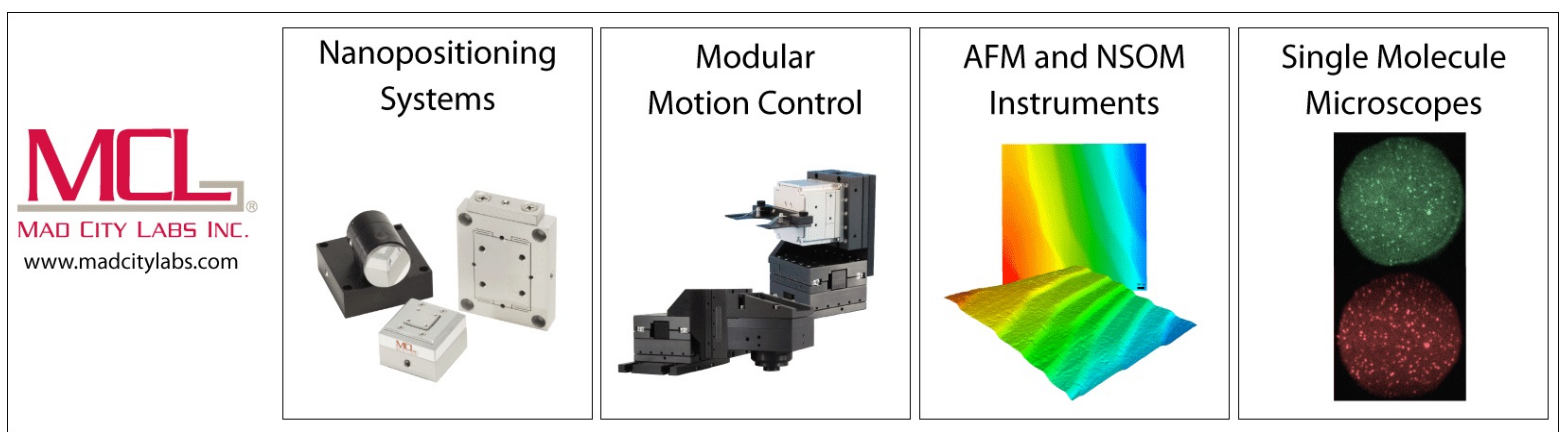




\title{
A simple method to measure the temperature and levitation height of devices rotating at cryogenic temperatures
}

\author{
Cite as: Rev. Sci. Instrum. 91, 045118 (2020); doi: 10.1063/5.0005498 \\ Submitted: 21 February 2020 - Accepted: 6 April 2020 • \\ Published Online: 27 April 2020
}

\begin{abstract}
Paolo de Bernardis, ${ }^{1,2}$ (D) Fabio Columbro, ${ }^{1,2}$ (D) Silvia Masi, ${ }^{1,2, a)}$ (D) Alessandro Paiella, and Giovanni Romeo
\end{abstract}

\author{
AFFILIATIONS \\ ${ }^{1}$ Dipartimento di Fisica, Sapienza Universitá di Roma, P.le A. Moro 2, 00185 Roma, Italy \\ ${ }^{2}$ INFN, Sezione di Roma, P.le A. Moro 2, 00185 Roma, Italy \\ ${ }^{3}$ Istituto Nazionale di Geofisica e Vulcanologia, Via di Vigna Murata 605, 00143 Roma, Italy
}

a) Author to whom correspondence should be addressed: silvia.masi@roma7.infn.it

\begin{abstract}
We describe a simple system to measure the temperature and levitation height of levitating cryogenic devices in rotation. Devices of this kind are the half-wave-plates rotating on superconducting magnetic bearings used in several cryogenic polarimeters for the cosmic microwave background. The temperature measurement is important to monitor the radiative background and potential systematic effects in the polarimeter. In our implementation, the temperature sensor is a thermistor, physically mounted on the rotating device. The sensor is biased with an AC current, which is transferred from the stationary electronics to the rotating device via capacitive coupling. The levitation height sensor is a network of capacitors, similar to the one used for the capacitive coupling of the thermistor. We describe the optimization of the readout system and its performance, which has been tested on a room-temperature prototype. We show that this system reaches an accuracy better than $3 \%$ for the measurement of the thermistor resistance and an accuracy of $\sim 10 \mu \mathrm{m}$ for the measurement of its levitation height.
\end{abstract}

Published under license by AIP Publishing. https://doi.org/10.1063/5.0005498

\section{INTRODUCTION}

Modern measurements of Cosmic Microwave Background $(\mathrm{CMB})$ polarization ${ }^{1}$ use large-throughput arrays of transition edge sensors (TESs) ${ }^{2-5}$ or kinetic inductance detectors (KIDs) ${ }^{6-9}$ operating in a cryogenic environment. The detectors are cooled to sub-K temperature to maximize their sensitivity, and the optical components of the polarimeter are also cooled to reduce the radiative loading on the detectors and minimize systematic effects. A Stokes polarimeter uses a rotating half-wave-plate (HWP) and a polarizer to modulate the linearly polarized component. The HWP temperature should also be minimized. This is very important, in particular, for space-based experiments (such as LiteBIRD ${ }^{10}$ ), where the thermal emission of the HWP can load the detectors, increase their photon-noise budget (basically limited by the CMB brightness), and produce modulation-synchronous effects (see Refs. 11 and 12 for a discussion). For these reasons, the temperature of the HWP should be kept lower than 10-20 K, depending on the frequency of operation of the detectors. Similar arguments with relaxed requirements are applicable to balloon-borne (such as LSPE-SWIPE ${ }^{13}$ ) and ground-based (such as QUBIC ${ }^{14}$ ) experiments. Superconducting magnetic bearings provide a nearly frictionless, fast rotation of the HWP, perfectly compatible with the cryogenic implementation of the Stokes polarimeter. ${ }^{15-18}$ The HWP assembly levitates and rotates in the magnetic field produced by the interaction of a permanent magnet in the rotor and a set of static superconducting bulks, reaching a mechanical rotation speed $f_{o} \sim$ a few $\mathrm{Hz}$ and modulating the polarized signal at $\sim 4 f_{o}$.

In this configuration, however, there is no mechanical contact between the HWP and the rest of the system. Any power dissipation (for example, due to eddy currents) or absorbed power (for example, due to the radiative load from the external environment) raises the 
temperature of the rotating assembly and the HWP, which can cool down only radiatively. At low temperatures, radiative cooling of the HWP is not effective, so the power dissipation on the rotor should be carefully minimized. In Fig. 1, we show the results of a simulation, performed with Comsol Multiphysics, ${ }^{19}$ of the temperature of a rotating metal-mesh HWP mounted on an aluminum rotor and surrounded by a $5 \mathrm{~K}$ environment. The simulation allows for thermal gradients in the plastic HWP (the conductivity of the very thin metal meshes is neglected). The temperature is monitored in the center of the HWP, while the gradient within the HWP itself is negligible $(\lesssim 10 \mathrm{mK})$. We assume $0.02 \mathrm{HWP}$ emissivity, flat in the relevant frequency band, while we assume to have blackened (emissivity $\sim 0.5$ ) the cryostat-side of the aluminum ring to favor cooling. The heating power comes from the radiation of the surrounding environment (2.7 K sky and $5 \mathrm{~K}$ cryogenic system) and from the eddy currents produced by the driving coil currents. With these assumptions, the total expected heat load is $\sim 0.1 \mathrm{~mW}$, evenly distributed over the edge of the aluminum ring. We also considered more pessimistic cases $(0.2 \mathrm{~mW}$ and $0.5 \mathrm{~mW}$, see Ref. 20 for details). From Fig. 1, it is evident that such small power inputs can produce a HWP temperature rise of a few $\mathrm{K}$ to tens of $\mathrm{K}$, resulting in an increased radiative load on the detectors, photon noise, and spurious synchronous signals.

For this reason, it is very important to monitor the temperature of the HWP using precise measurements. In the case of a contactless rotor, one way to measure the temperature of the HWP is by monitoring its thermal emission. There are two basic ways of implementing such a measurement. The first one is to measure directly the emission of the optical device, provided it is sufficiently emissive at the measurement wavelength and in the temperature range of interest. Since the temperature of the HWP is expected to be $<20 \mathrm{~K}$, its thermal emission will be very weak for wavelengths much shorter than $150 \mu \mathrm{m}$. Monitoring the emission at wavelengths longer than $150 \mu \mathrm{m}$ is also difficult, since the materials currently used in HWPs for $\mathrm{CMB}$ measurements (sapphire or polyethylene embedding thin

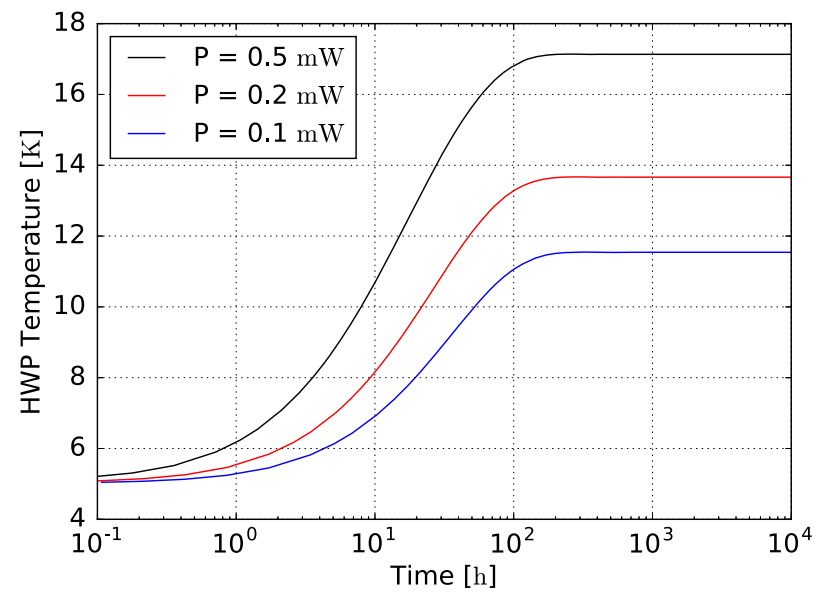

FIG. 1. Simulation of the temperature of a levitating rotating metal-mesh HWP ( 300 $\mathrm{mm}$ diameter, which is the range of interest for the HWP of the LiteBIRD experiment ${ }^{10,21}$ ), radiatively heated with three different levels of input power, starting from a base temperature of $5 \mathrm{~K}$. See the text for details. metal meshes) have low emissivity at wavelengths longer than $150 \mu \mathrm{m}$. In this case, it is difficult to modulate the thermal emission to be measured. The second approach is to blacken, e.g., two quarter sectors of the ring supporting the HWP optical device and make the other two quarter sectors reflective. The detector can look at the rotating ring, measuring the modulated signal produced alternating the emissive and reflective quarters of the ring. If the reflective sectors reflect a stable background, the amplitude of this signal will depend on the temperature of the support ring, which might be considered a good proxy of the temperature of the HWP. The blackened sectors of the ring will also help to cool down the rotating HWP assembly. The signal detected with a reasonable throughput $\left(0.2 \mathrm{~cm}^{2} \mathrm{sr}\right)$ and optical bandwidth (20\%) at a sensing wavelength of, e.g., $1.4 \mathrm{~mm}$ is in the $1-100 \mathrm{pW}$ range for temperatures between $3 \mathrm{~K}$ and $20 \mathrm{~K}$. For a precision measurement, sensitive detectors are required, especially at the lowest edge $(\sim 3 \mathrm{~K})$ of the temperature range.

Here, we describe a simpler, cost effective, reliable method to measure the temperature of the rotating assembly, avoiding the use of such a complex detection system. This is based on a regular thermistor, mounted on the rotating assembly. The thermistor is capacitively coupled to the steady readout system and biased with an AC current.

A simple extension of the method also allows for monitoring the distance of the rotor with respect to the stator along the rotation axis, i.e., its levitation height. This is also a very important parameter of the system, and since the coupling capacitor capacitance is a strong function of the distance between the plates, the proposed measurement system will also serve efficiently to this purpose.

In the following, we describe the measurement system, its optimization, and its performance. In Sec. II, we describe the capacitively coupled readout of the thermistor and of the rotor levitation height, including the general design and the optimization of the electronics. In Sec. III, we describe the results of a validation of the method, carried out using a room-temperature $600 \mathrm{~mm}$ diameter rotating disk as a stand-in for the cryogenic half-wave plate rotor. In Sec. IV, we summarize and discuss the results.

\section{THE CAPACITIVELY COUPLED THERMISTOR}

\section{A. Practical implementation}

The block diagram of the proposed system is shown in Fig. 2. A regular thermistor for cryogenic temperature measurements $\left[\mathrm{RuO}_{2}\right.$ resistor, CERNOX ${ }^{\mathrm{TM}}$ (Ref. 22), or similar, with resistance values ranging between $500 \Omega$ and $50 \mathrm{k} \Omega$ ] is mounted on the rotary assembly. Each contact of the thermistor is connected to one pole of a plane capacitor, i.e., a simple rectangular copper pad (10 mm wide $\times 50 \mathrm{~mm}$ long, see below) mounted on the rotating assembly. The other pole of the same capacitor is similar and is mounted on the stator. At a certain angle of the rotation of the HWP, the two poles of both capacitors will align one on top of the other, with a gap of $\sim 1 \mathrm{~mm}$ between the poles, thus creating two capacitors. Each capacitance can be computed as

$$
2 C=\epsilon_{0} \frac{A}{d} \sim 4.4 \mathrm{pF} .
$$

The steady pole of the first capacitor is connected to an AC voltage generator, while the steady pole of the second capacitor 


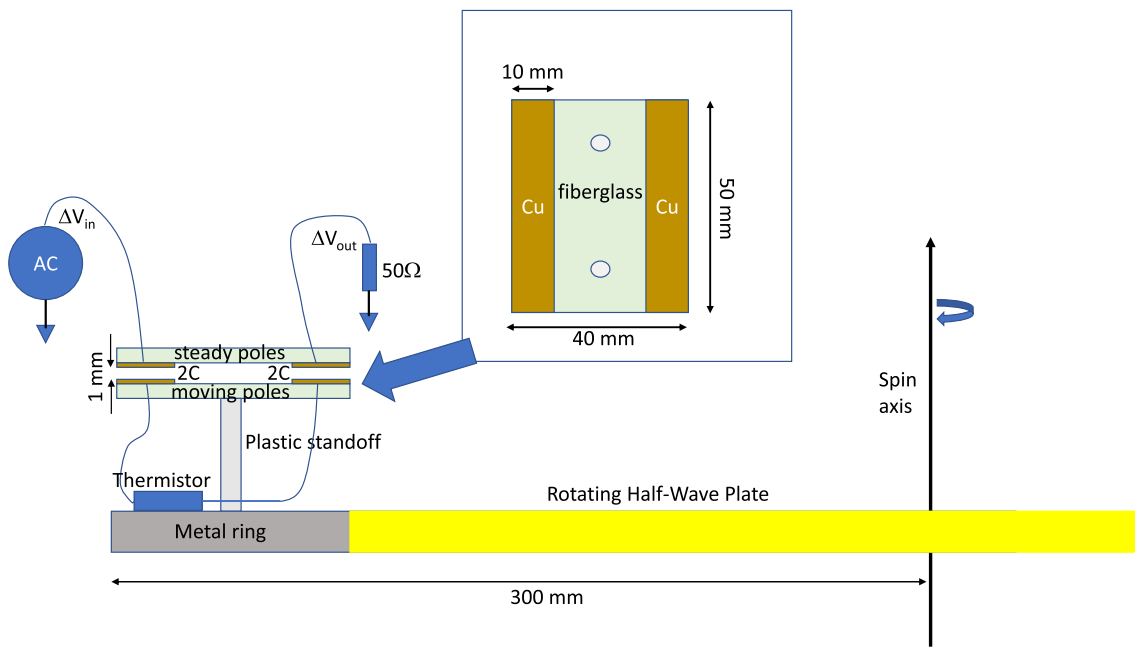

FIG. 2. Schematic diagram of the temperature and distance measurement concept. The dimensions refer to the room-temperature demonstration mockup (600 $\mathrm{mm}$ diameter) where the HWP was replaced by an inertially equivalent aluminum disk rotating on a low-friction ball-bearing.

is connected to a low-value load resistor $\left(R_{L} \sim 50 \Omega\right)$ closing the circuit to ground. When the poles are aligned, an AC current flows through the thermistor with an amplitude depending on its resistance $R$, which, in turn, depends on its temperature $[R=R(T)]$.

The sizing of the capacitor poles and their separation is a tradeoff between different needs. The distance between the moving and steady poles (nominally $1-2 \mathrm{~mm}$ ) is a tradeoff between the need of maximizing the capacitance and the need to operate in a relatively wide range of levitation heights and to cope with the small height drop expected when the rotor is released from its clamping device. We have to limit the global size of the sensor to accommodate it above the rotor ring and within the shields protecting the optical beam crossing the HWP. On the other hand, a large area of the poles would produce a large capacitance and thus a large transmitted signal. It would also produce a wider signal peak when the steady and moving poles align: this would make it easier to properly sample it and evaluate its maximum amplitude. For simplicity of implementation, we accommodated the steady poles of the input and output capacitors on the same printed circuit board (PCB); the same layout has been used for the moving poles. A large distance between the two poles on the same PCB would reduce the parasitic capacitance (see below). In our prototype, the best tradeoff was found for rectangular poles, $10 \mathrm{~mm}$ wide $\times 50 \mathrm{~mm}$ long, separated by a $20 \mathrm{~mm}$ gap.

Parasitic capacitances are present in the setup sketched in Fig. 2. Since two poles of different capacitors are present on the same PCB, there is a parasitic capacitance between these poles, $C_{p B}$ for the steady poles and $C_{p T}$ for the moving poles. Its effect is to transfer part of the bias signal directly from the input to the output without biasing the thermistor. The $20 \mathrm{~mm}$ distance between the poles reduces this parasitic capacitance to $\sim 1 \mathrm{pF}$, assuming $\epsilon_{r}=4.4$ for the PCB material. This applies to both the input and output poles of the steady capacitors and to the input and output poles of the moving capacitors, where $C_{p T}$ is in parallel to the thermistor. In an optimized implementation, we can reduce $C_{p T}$ and $C_{p B}$ by a factor of $\sim 4$ using separate standoffs for the input and output poles. In any case, in the analysis of the capacitively coupled thermistor, $C_{p B}$ and $C_{p T}$ are not negligible with respect to $2 C$.

From Eq. (1), it is immediately evident that the capacitance is a strong function of the distance between the poles. Hence, the amplitude and phase of the signal transferred to the rotating assembly via the capacitors will be a function of the levitation height. This means that we have to disentangle the two effects (changes in thermistor resistance and changes in levitation height). This can be done by doubling the moving sets of capacitor poles. We will use one set of capacitor poles to bias the thermistor connected between them (thermistor network) and the other one to bias a metal wire, with negligible resistance, connected between the two moving poles (reference network). The amplitude of the signal of the reference network depends only on the levitation height, allowing a measurement of it and a correction of measurements of the thermistor network. We discuss these issues in Sec. III.

\section{B. Basic circuit analysis and optimization}

The equivalent circuit of the capacitively coupled thermistor is reported in Fig. 3.

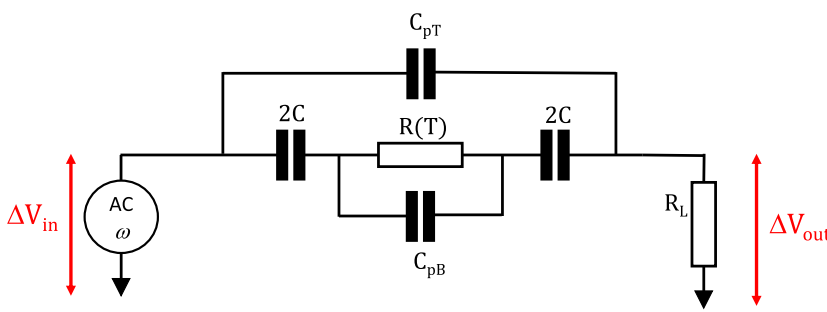

FIG. 3. Equivalent circuit of the temperature measurement. The excitation $\Delta V_{\text {in }}$ is produced by a voltage generator at frequency $f=\omega /(2 \pi)$. The rotating capacitors have capacitance $2 C ; R(T)$ is the thermistor resistance; $C_{D T}$ is the parasitic capacitance between the steady poles of the two capacitors; $C_{D B}$ is the parasitic capacitance between the rotating poles of the two capacitors; and $R_{L}$ is the load resistor. 
The transfer function for the circuit in Fig. 3 can be written as

$$
\frac{\Delta V_{\text {out }}}{\Delta V_{\text {in }}}=\frac{1}{1+\frac{Z}{R_{L}}},
$$

where $\Delta V_{\text {out }}$ and $\Delta V_{\text {in }}$ are the measurement and drive voltage, respectively, $R_{L} \sim 50 \Omega$ is the load resistor, and $Z$ is the combined impedance of the network of horizontal components in Fig. 3. We obtain

$$
\begin{gathered}
\operatorname{Re}(Z)=\frac{R(T)}{\left(1+C_{p T} / C\right)^{2}+\omega^{2} R^{2}(T) C_{p}^{2}}, \\
\operatorname{Im}(Z)=-\frac{1}{\omega C} \frac{\left(1+C_{p T} / C\right)+\omega^{2} R^{2}(T)\left(C+C_{p B}\right) C_{p}}{\left(1+C_{p T} / C\right)^{2}+\omega^{2} R^{2}(T) C_{p}^{2}}, \\
C_{p}=C_{p B}\left(1+C_{p T} / C\right)+C_{p T},
\end{gathered}
$$

where $\omega=2 \pi f$ is the angular frequency of the drive signal.
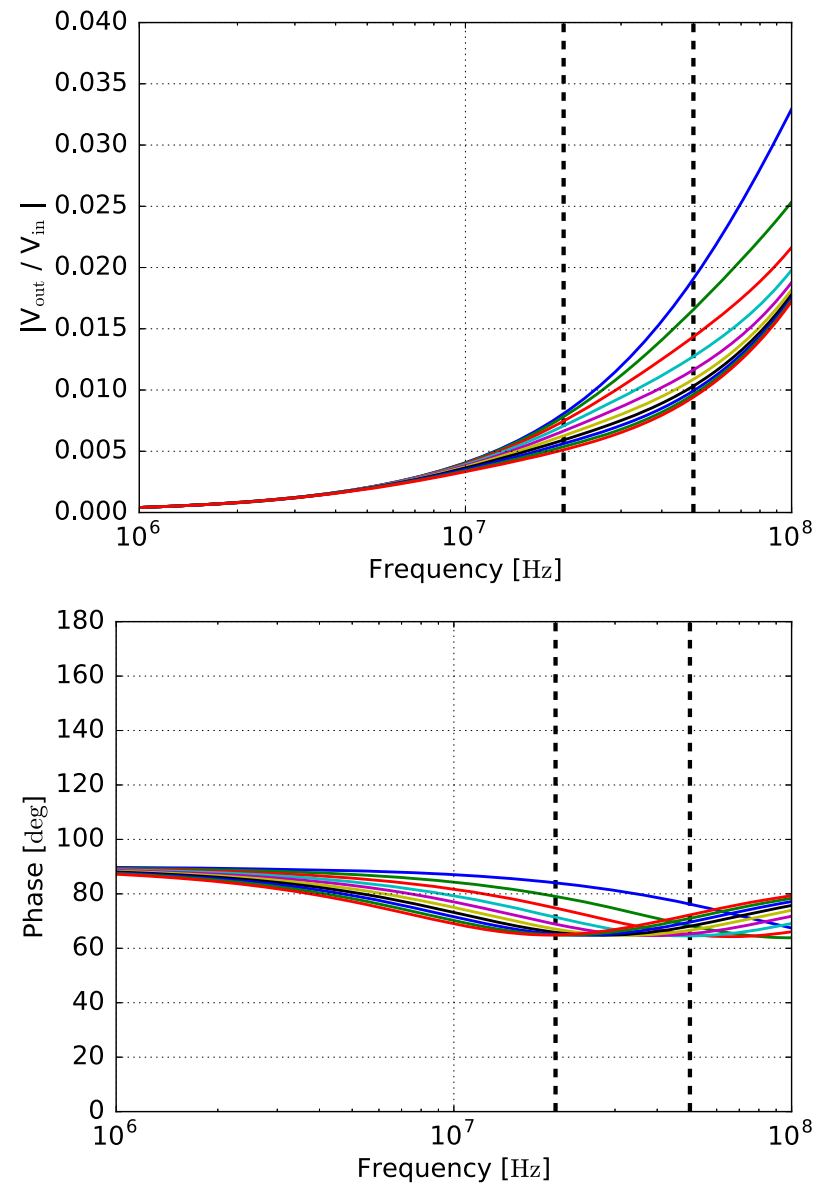

FIG. 4. Modulus (top) and phase shift (bottom) of the transfer function of the readout circuit for thermistor values between $1 \mathrm{k} \Omega$ (top line) and $10 \mathrm{k} \Omega$ (bottom line) in $1 \mathrm{k} \Omega$ steps. The optimal frequency range of operation (significant and roughly equalized response to thermistor resistance variations) is approximately bracketed by the two dashed lines.
The modulus and phase shift of the transfer function are plotted in Fig. 4 for a thermistor resistance $R(T)$ ranging from $1 \mathrm{k} \Omega$ to $10 \mathrm{k} \Omega$, a coupling capacitance $2 C=1 \mathrm{pF}$ (a value expected for a distance of $\sim 4 \mathrm{~mm}$ between the steady and moving poles in our prototype), a parasitic capacitance between the steady poles of the two capacitors $C_{p T}=0.3 \mathrm{pF}$, and a parasitic capacitance between the rotating poles of the two capacitors $C_{p B}=0.3 \mathrm{pF}$ (as expected using separate standoffs for the two poles).

From Fig. 4, it is evident that for the typical values of the components considered here, the optimal excitation frequency is in the $20-50 \mathrm{MHz}$ range, where the output signal excursion is significant and roughly equalized. This frequency range also has the advantage of being significantly higher than the typical bias frequency of frequency-domain multiplexed TES $(\sim 1 \mathrm{MHz})$ and significantly lower than the typical bias frequency of $\operatorname{KID}(\sim 1 \mathrm{GHz})$ so that the crosstalk between this signal and CMB detector signals is minimized.

The expected non-linear relation between the resistance of the thermistor and the output signal is shown in Fig. 5.

With an excitation amplitude of $1 \mathrm{~V}_{\mathrm{rms}}$, the output signal is in the range of $10 \mathrm{mV}_{\mathrm{rms}}$. However, in the range of temperatures of interest here, the rms voltage across the thermistor must be limited below $0.01 \mathrm{~V}_{\text {rms }}$ to avoid self-heating of the thermistor. This requires an excitation amplitude of the capacitor-thermistor network smaller than $1 \mathrm{~V}_{\text {rms }}$, as shown in Fig. 6. For example, at $25 \mathrm{MHz}$, for an excitation amplitude of $50 \mathrm{mV}_{\text {rms }}$, we have a signal across the thermistor of $\sim 10 \mathrm{mV}_{\text {rms }}$ and a power dissipation in the thermistor of $\sim 100 \mathrm{nW}$. This adds negligible heating in the thermistor and in the rotor. Note also that the biasing is intermittent, further reducing the heat load. The small excitation, however, results in very small (order of $0.3 \mathrm{mV}_{\text {rms }}$ ) output signals across the load resistor of Fig. 3. For this reason, a custom readout scheme has been developed, as described below.

\section{Excitation and readout circuit}

In the simplest implementation, the excitation signal is obtained from a $25 \mathrm{MHz}$ digital clock, which is filtered by a resonant circuit to obtain a sine-wave. We prefer to remove the higher

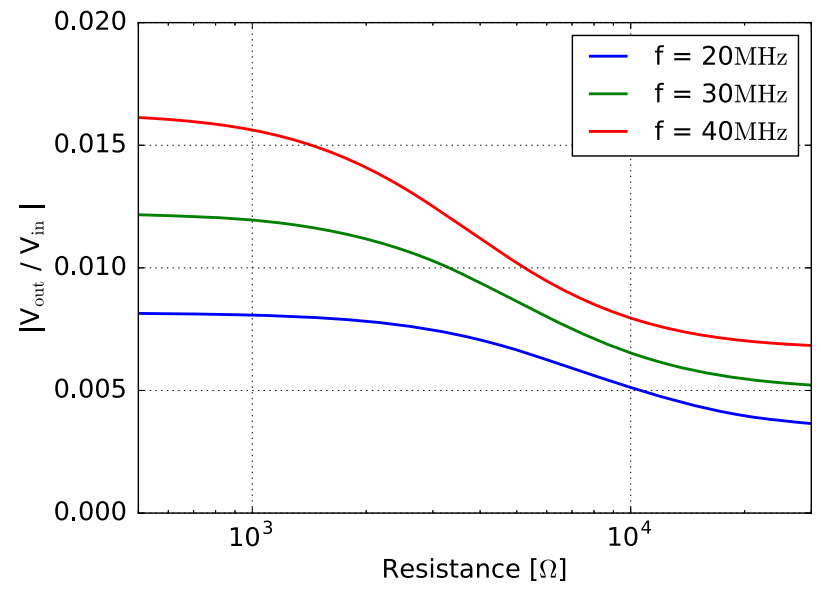

FIG. 5. Expected output voltage as a function of the thermistor resistance for excitation frequencies $f=20 \mathrm{MHz}, 30 \mathrm{MHz}$, and $40 \mathrm{MHz}$ (bottom to top lines). 


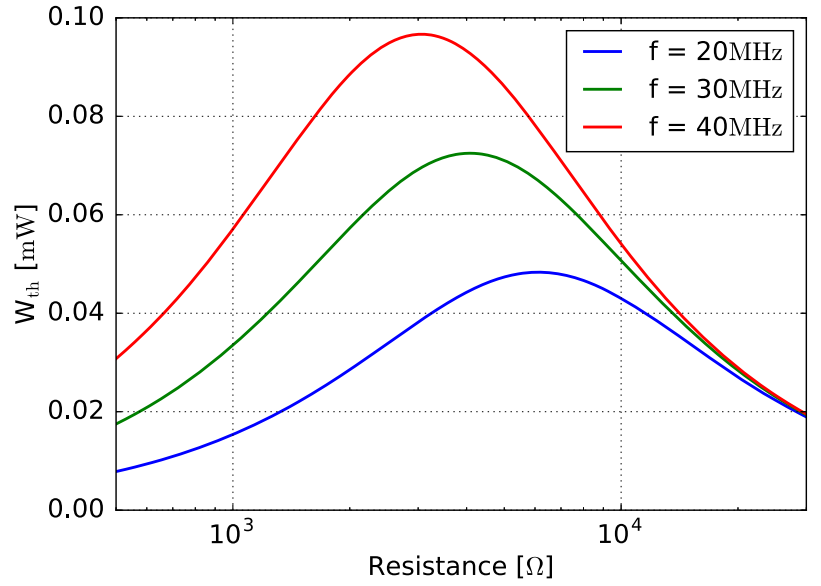

FIG. 6. Expected power dissipated in the thermistor, as a function of the thermistor resistance, for excitation frequencies $f=20 \mathrm{MHz}, 30 \mathrm{MHz}$, and $40 \mathrm{MHz}$ (bottom to top lines) for a $1 \mathrm{~V}_{\mathrm{rms}}$ excitation amplitude. The power scales as the square of the excitation voltage and must be reduced to avoid self-heating of the thermistor.

harmonics present in the original square-wave, since they produce large spikes through the capacitor-thermistor network of Fig. 3, which are difficult to model due to the presence of stray capacitance and uncertainties in the high frequency gain of the active components in the following signal recovery chain. Having a pure tone would also minimize the crosstalk between this signal and the signals from the $\mathrm{CMB}$ detectors. The sine-wave signal is equally split between two lines using a simple SMA (SubMiniature version A) tee adapter. One line is used to bias the capacitively coupled thermistor, and the other is read by an amplitude monitor. The tone supplying the capacitively coupled thermistor is reduced in amplitude by means of a $-20 \mathrm{~dB}, 50 \Omega$ SMA coaxial attenuator to obtain the excitation signal $\Delta V_{\text {in }}$ and connected via a $50 \Omega, 2 \mathrm{~m}$ long stainless steel coaxial cable to the capacitor-thermistor network. This results in an excitation across the thermistor $\Delta V_{t h} \sim 10 \mathrm{mV}_{\text {rms }}$. The signal across the load resistor is connected via a $50 \Omega, 2 \mathrm{~m}$ long coaxial cable to the input of a low-noise amplifier, based on a Mini-Circuits MAR-8 MMIC ${ }^{23}$ module, with gain $\sim 30 \mathrm{~dB}$. The output is low-pass filtered and sent to an RF-to-rms converter based on an Analog
Devices AD8361 ${ }^{24}$ chip. The DC output of the AD8361 is amplified by a factor of 10 and digitized. In the amplitude monitor, a $-10 \mathrm{~dB}$, $50 \Omega$ SMA coaxial attenuator adapts the signal level to the input of a second RF-to-rms converter, used to monitor any change in the excitation amplitude. The block-diagram of the readout electronics is shown in Fig. 7.

The output of this readout circuit has a significant offset due to the parasitic capacitance and the rms value of the noise of the thermistor and amplifier but is usable anyway if the noise level is stable (i.e., if external noise and radio-frequency interference (RFI) sources do not couple significantly to the capacitor-thermistor network).

In Fig. 8, we plot the measured response of the readout circuit to different values of the thermistor resistance. The measurement has been carried out by replacing the thermistor with different fixedvalue precision resistors.

In the previous analysis, we have neglected the effect of resistive losses in the coaxial cables. Low heat conductivity stainless steel coaxial cables, few meters long, have a high resistance of the order of a few $100 \Omega$. This is of the same order of the impedance of the coupling capacitors at our readout frequency and of the order of the resistance of the thermistor at the lowest temperatures. Moreover, its value changes with temperature from room-temperature to cryogenic temperatures, so it cannot be easily calibrated out using room-temperature measurements alone. If not properly taken into account, the change in resistive losses can produce a systematic error in the measurement of the temperature of $10 \%-20 \%$ in the low-edge of the temperature range. On the other hand, at variance with $\sim$ DC measurements, 4-wire measurements are difficult to carry out at these frequencies due to the need of terminating all the coaxial lines with $50 \Omega$ matched loads, implying that the readout lines cannot be of high impedance. For all these reasons, our approach is to use an additional reference network, measuring a stable capacitor, with the same capacitance as the moving capacitors. The stable capacitor is read by means of a couple of coaxial lines identical to the ones used for the temperature and levitation height measurements and using the same generator and RF level meter used for the other measurements. The output signal from this channel will depend only on the losses in the coaxial cables and can be used to normalize out the effect of their temperature-driven changes.

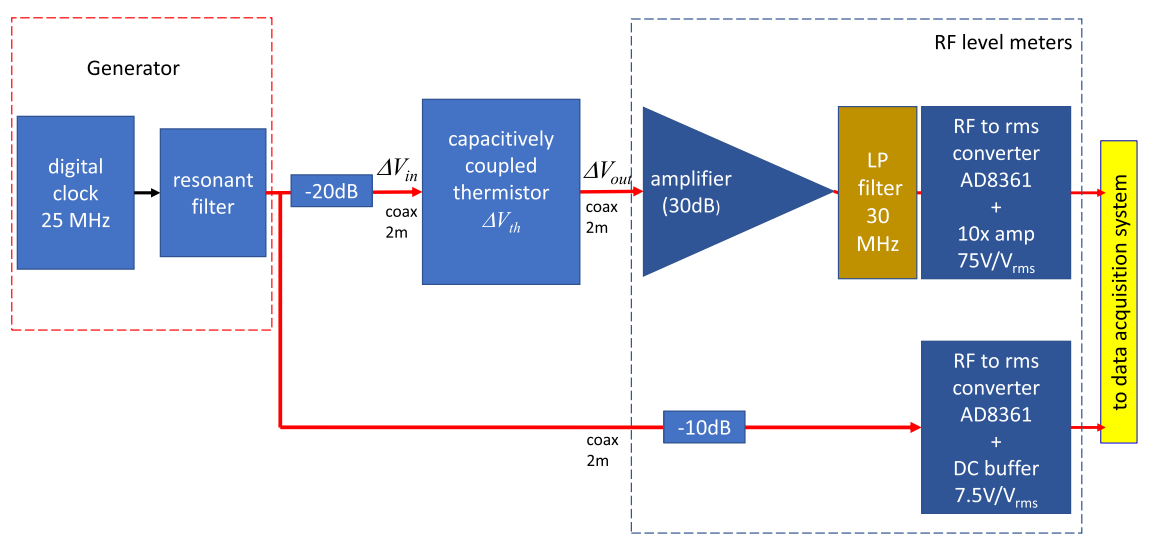

FIG. 7. Block-diagram of the simplest excitation and readout scheme. All components other than the capacitively coupled thermistor are shielded and operated at room-temperature. The thermistor and its coupling capacitors operate at cryogenic temperatures (few K). 


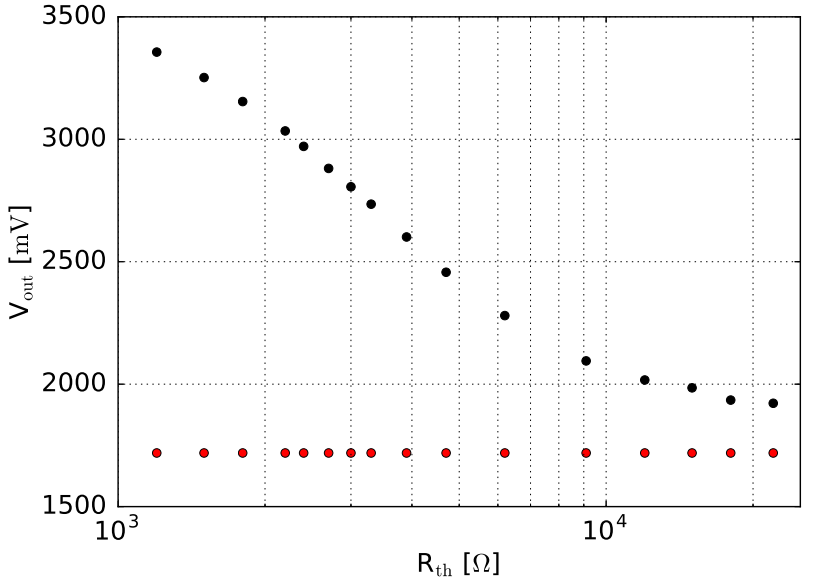

FIG. 8. Measured response of the readout circuit shown in Fig. 7 to different values of the thermistor resistance (black dots). For these measurements, we replaced the thermistor with a set of fixed-value precision resistors. The measurement frequency is $25 \mathrm{MHz}$. The red dots represent the measured values of the excitation amplitude monitor output. If the thermistor is a CERNOX ${ }^{\mathrm{TM}}$ model CX-1050, resistance values between $1 \mathrm{k} \Omega$ and $10 \mathrm{k} \Omega$ correspond to temperatures between $\sim 10 \mathrm{~K}$ and $\sim 2 \mathrm{~K}$, which is the range of interest for the HWP of the LSPE ${ }^{13}$ and QUBIC ${ }^{25}$ experiments.

The output noise of the readout circuit depends on the Johnson noise of the thermistor, on the noise of the amplifier, and on the electromagnetic interference (EMI) on the capacitive sensor. For a $50 \Omega$ load resistor, the Johnson voltage noise is $\sqrt{4 k_{B} T R} \sim 1 \mathrm{nV} / \sqrt{\mathrm{Hz}}$. Given the small value of the source resistance, the current noise of the amplifier is negligible, while the voltage noise of the amplifier is $\sim 1 \mathrm{nV} / \sqrt{\mathrm{Hz}}$. Over a bandwidth $\Delta f \sim 30 \mathrm{MHz}$, with a gain $G \sim 30$, the standard deviation of the output of the amplifier is rms $\sim 0.2$ $\mathrm{mV}_{\text {rms. }}$. The RF-to-rms converter operates with a time constant $\tau \sim$ $20 \mu \mathrm{s}$ and with a gain $G_{c} \sim 7.5 \mathrm{~V} / \mathrm{V}_{\mathrm{rms}}$, and an additional gain $G_{a}=$ 10 is provided by the output amplifier. The minimum fluctuation of the output of the readout circuit is thus $\sigma_{\text {out }}=G_{c} G_{a} \times r m s / \sqrt{2 \tau \Delta f} \sim$ $0.5 \mathrm{mV}_{\text {rms. }}$.

The average value of the output due to the bias signal is of the order of several hundreds of $\mathrm{mV}_{\mathrm{rms}}$ to $\sim 2 \mathrm{~V}_{\mathrm{rms}}$, resulting in an expected signal-to-noise ratio much larger than 100, if the interference is negligible.

We have tested the previous forecast acquiring the output of the readout with a 24-bit analog-to-digital (A/D) converter. The measured rms fluctuation is consistent with the expected value when the capacitor network is well shielded in a grounded metal box. Such a configuration is representative of the operation of the sensor inside the metal cryostat of a CMB polarimeter.

When operated in the laboratory, outside the metal shielding, the system is sensitive to the position with respect to the rotator and the environment and also to cell-phones and computer-generated electromagnetic interference (EMI). These effects limit the performance of the system in the measurements reported below, which, nonetheless, reach an acceptable performance level.

The improved performance of the readout and reduced sensitivity to EMI might be obtained by reducing the bandwidth at the amplifier output, e.g., replacing the low-pass filter in Fig. 7 with a resonant filter. In order to avoid thermally driven mismatches between the filter response and the generator frequency, a lockin demodulation might be used. We leave this to future developments, since even for the simple readout circuit described above, the readout noise is not the limiting factor, as we show below.

\section{MEASURED PERFORMANCE}

We tested the performance of the measurement method using a room-temperature mock-up, where the levitating rotor is replaced by a $600 \mathrm{~mm}$ diameter aluminum disk rotating on a low-friction ball-bearing, and the thermistor has been simulated using resistors with values spanning the expected range $R_{t h} \sim 1 \mathrm{k} \Omega-10 \mathrm{k} \Omega$. The rotor driver, based on steady coils pushing or pulling on permanent magnets on the disk, and the strong levitation magnet of the superconducting magnetic bearing are the same as for the cryogenic device. As explained at the end of Sec. II A, we have a reference network for levitation height measurements and a thermistor network for temperature measurements. In the following, we describe the measurements we carried out with the room-temperature mock-up for both networks.

\section{A. Distance measurements}

The capacitance $2 C$ of the coupling capacitors depends on the distance between the steady and moving capacitor poles [Eq. (1)], which depends directly on the levitation height of the rotor. In the reference network, the two coupling capacitors are simply connected in series by a short conductor replacing the thermistor and connecting the two rotating poles. As a result, the amplitude and phase of the transmitted signal depend only on the levitation height. During the rotation of the rotor, the steady capacitor poles will sequentially face the moving poles of the thermistor network capacitors and the moving poles of the reference network capacitors. Hence, the output of the readout chain will monitor in close sequence the thermistor resistance and the distance between the poles.

In Fig. 9, we report sample records of the raw readout output for a system with four networks. Here, two thermistors with resistances of $10 \mathrm{k} \Omega$ and $4.7 \mathrm{k} \Omega$, spaced by $180^{\circ}$ in azimuth, are mounted on the rotor. Each thermistor network is followed by its reference network. The measurement has been repeated for different values of the distance $h$ between the moving capacitor poles and the steady capacitor poles while the rotor is spinning at $\sim 40 \mathrm{rpm}$. Note that at this spin rate, the signal peak is $\sim 75 \mathrm{~ms}$ wide. This is much longer than the integration time of the RF-to-rms converter $(\sim 20 \mu \mathrm{s})$, thus allowing for much faster spin rates without any significant degradation of the peak height.

The data demonstrate that the reference network allows for a good measurement of the distance between the poles. If we assume a distance between the capacitor poles of $1 \mathrm{~mm}$, the system has a resolution $d V / d h \sim 1.6 \mathrm{~V} \mathrm{~mm}^{-1}$, while for a distance of $2 \mathrm{~mm}$, the resolution is $d V / d h \sim 0.75 \mathrm{~V} \mathrm{~mm}^{-1}$.

Note that the measured rms fluctuation of the readout output $\sigma_{M}$ is several $\mathrm{mV}$ rms larger than the one expected from the Johnson and amplifier noise alone. This means that significant perturbations arise from the unshielded operation and the stray capacitance variations with respect to the surrounding environment. 


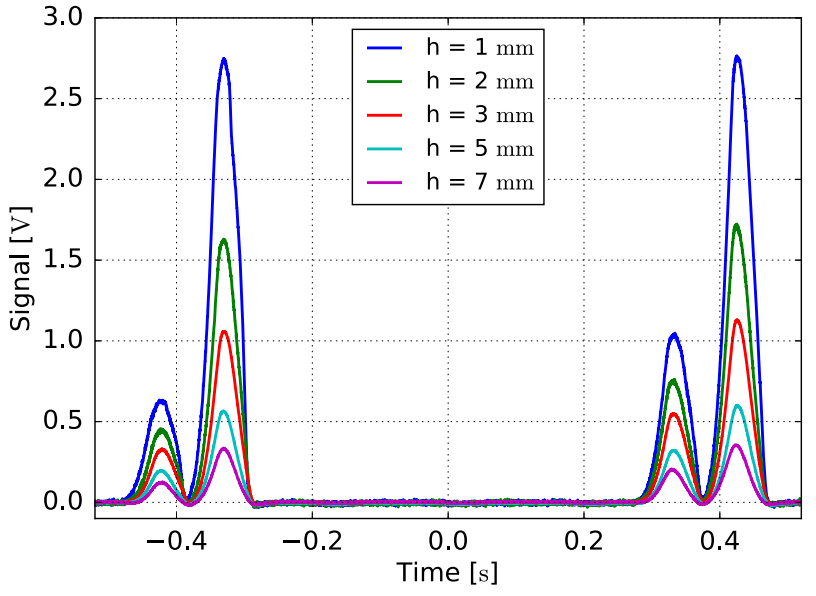

FIG. 9. Sample records of the readout output while the rotor was spinning at $40 \mathrm{rpm}$. The four peaks (left to right) correspond to the first thermistor network $\left(R_{t h}=10 \mathrm{k} \Omega\right)$, its reference capacitor, a second thermistor network $\left(R_{t h}=4.7 \mathrm{k} \Omega\right)$, and its reference capacitor. The reference capacitors provide an effective way to estimate the distance between the capacitor poles, which depends on the vertical position of the levitating rotor.

Using anyway the measured noise, we can also compute the error in the measurement of the levitation height,

$$
\sigma_{h}=\frac{d V}{d h} \sigma_{M}
$$

We find that variations of the height as small as $\sigma_{h} \sim 6 \mu \mathrm{m}$ and $\sigma_{h} \sim 13 \mu \mathrm{m}$ for distances $h$ of $1 \mathrm{~mm}$ and $2 \mathrm{~mm}$, respectively, can be detected reading the reference capacitive networks.

Note that, in principle, any tilt of the rotor (which might introduce systematic effects in high precision applications; see, e.g., Ref. 26) could be dynamically sampled by replicating the readout chain and the assembly of static poles in three replicas separated by $120^{\circ}$ in azimuth.

\section{B. Temperature measurements}

The thermistor network is sensitive to both temperature changes and levitation height changes. The distance measurements from the reference network described in Sec. III A can thus be used to correct the effect of distance fluctuations in the measurements of the thermistor network.

In order to check the effectiveness of the method, we normalize the peak signal of each thermistor network to the peak signal of its reference network and plot in Fig. 10 their ratios. Regardless of the distance between the capacitor poles, the ratio remains approximately constant, confirming the effectiveness of the correction method.

The measured output signal noise $\sigma_{M}$ is readily converted into an error in the temperature measurement, once the $R_{t h}(T)$ characteristic of the thermistor is defined,

$$
\Delta T=\frac{d T}{d R} \sigma_{R}=\frac{d T}{d R} \frac{d R}{d V} \sigma_{M},
$$

where the first derivative is estimated from the calibration curve of the thermistor $R_{t h}(T)$, while the second derivative is estimated

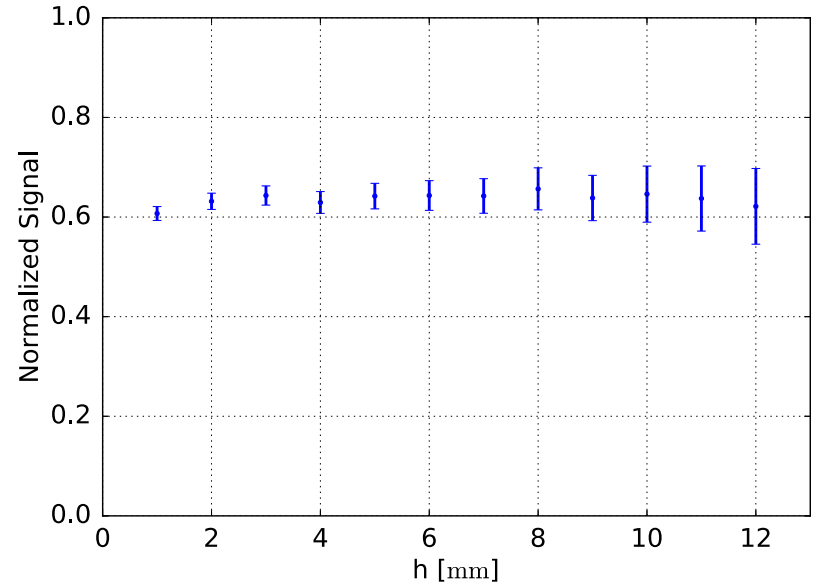

FIG. 10. Ratio between the peak signals of two thermistor networks $\left(R_{t h}=4.7 \mathrm{k} \Omega\right.$ and $R_{t h}=10 \mathrm{k} \Omega$ ), each normalized to the peak signal of its reference network, plotted for a wide range of distances between steady and moving capacitor poles.

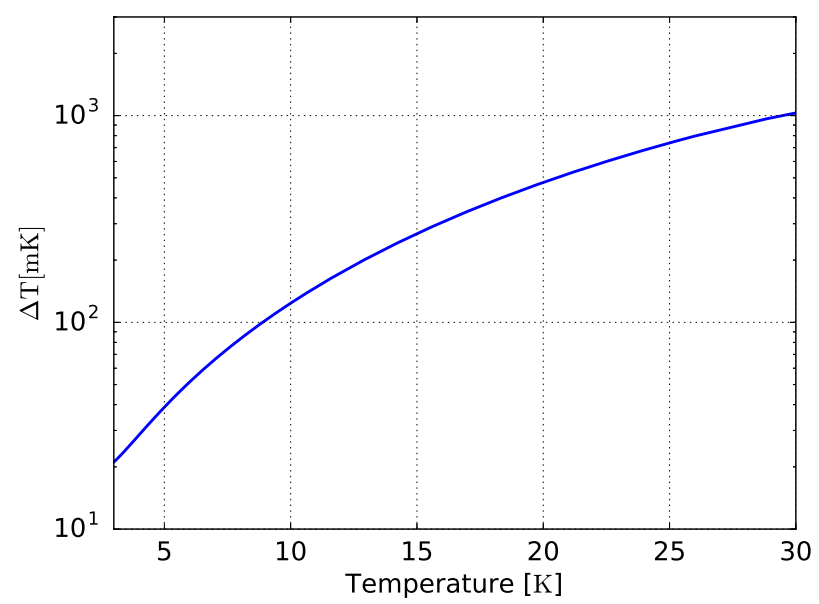

FIG. 11. Error on the measured temperature, estimated for a CX-1050 sensor read through the readout circuit described in the text.

from the calibration data $V\left(R_{t h}\right)$ of Fig. 8. In Fig. 11, we plot $\Delta T$ for a CERNOX ${ }^{\mathrm{TM}}$ CX-1050 temperature sensor. Since the measured $\sigma_{M}$ does not depend on the value of the thermistor resistance, the behavior of $\Delta T$ vs rotor temperature is due to the combination of the thermistor resistance $R_{t h}(T)$ becoming steeper for lower temperatures and the $V\left(R_{t h}\right)$ of the readout circuit mildly decreasing for the correspondingly larger resistances. The relative error remains smaller than $3 \%$ in the entire temperature range of interest.

\section{CONCLUSIONS}

We have designed, built, and tested a device able to sense the temperature and position of a levitating rotor during its fast rotation at cryogenic temperatures. The measurement is based on regular 
thermistors, mounted on the rotor, periodically and briefly biased by a high-frequency AC current through a capacitive network.

We have demonstrated the correct operation of the capacitive readout in a room-temperature real-world environment. We included the electromagnetic interference of the rotor driver coils and of the levitation magnet and even variable capacitive coupling to the surrounding environment. The latter will arguably be absent in the cryogenic operation inside a grounded cryostat shell. The presence of varying capacitive coupling toward the surrounding environment results in increased output fluctuations with respect to the ones due to resistors and amplifier noise, but the resulting noise is sufficiently small to permit measurements of the temperature at precisions required for upcoming experiments.

An alternative inductive coupling between the rotating thermistor and the static readout system, while, in principle, possible and effective, would be severely affected by the magnetic interference from the driving coils.

We do not expect any change in the performance of the measurement setup when cooled to cryogenic temperatures. The coaxial cables we used have the same dimensions and length as those that will be used in the cryogenic setup, and we do not use any electronic component near the capacitive networks that are intrinsically temperature-independent.

Using a CX-1050 CERNOX ${ }^{\mathrm{TM}}$ thermistor, the error in the measured resistance converts into an error in the temperature smaller than $3 \%$ for the $2-30 \mathrm{~K}$ temperature range of interest for the levitating HWPs of cryogenic CMB polarimeters.

Reference capacitive networks read through the same circuit allow for precision measurements of the levitation height of the rotor with a resolution of $\sim 10 \mu \mathrm{m}$. This provides an important diagnostic of the dynamics of the rotor and allows for corrections of the thermistor resistance measurement fluctuations due to any variation in levitation height.

The proposed monitoring system can be implemented in cryogenic CMB polarimeters with modest impact on the cryogenic system and good scalability: it needs only two coaxial cables for the each static set of capacitor poles plus two coaxial cables for monitoring the resistive losses; it produces negligible dissipation on the HWP rotating assembly $(<0.1 \mu \mathrm{W}$ per thermistor). The size of the coupling capacitor poles can be tailored to fit any HWP support ring, if this is wider than $\sim 1 \mathrm{~cm}$. The system operates correctly for any reasonable HWP rotation speed. We plan to install it in the forthcoming SWIPE instrument of LSPE ${ }^{13}$ and possibly in the MHFT (Medium High Frequency Telescope) instrument of LiteBIRD.

\section{ACKNOWLEDGMENTS}

This research was supported by the Italian Space Agency (ASI: LSPE and LiteBIRD contracts) and by the Italian National Institute for Nuclear Physics (INFN-LSPE activity).

The data that support the findings of this study are available from the corresponding author upon reasonable request.

\section{REFERENCES}

${ }^{1}$ D. Baumann and H. V. Peiris, Adv. Sci. Lett. 2, 105 (2009).

${ }^{2}$ K. D. Irwin and G. C. Hilton, "Transition-edge sensors," in Cryogenic Particle Detection, edited by C. Enss (Springer Berlin Heidelberg, Berlin, Heidelberg, 2005), pp. 63-150.
${ }^{3}$ C. M. Posada, P. A. R. Ade, A. J. Anderson, J. Avva, Z. Ahmed, K. S. Arnold J. Austermann, A. N. Bender, B. A. Benson, L. Bleem, K. Byrum, J. E. Carlstrom, F. W. Carter, C. Chang, H.-M. Cho, A. Cukierman, D. A. Czaplewski, J. Ding, R. N. S. Divan, T. de Haan, M. Dobbs, D. Dutcher, W. Everett, R. N. Gannon, R. J. Guyser, N. W. Halverson, N. L. Harrington, K. Hattori, J. W. Henning, G. C. Hilton, W. L. Holzapfel, N. Huang, K. D. Irwin, O. Jeong, T. Khaire, M. Korman, D. L. Kubik, C.-L. Kuo, A. T. Lee, E. M. Leitch, S. L. Escudero, S. S. Meyer, C. S. Miller, J. Montgomery, A. Nadolski, T. J. Natoli, H. Nguyen, V. Novosad, S. Padin, Z. Pan, J. E. Pearson, A. Rahlin, C. L. Reichardt, J. E. Ruhl, B. Saliwanchik, I. Shirley, J. T. Sayre, J. A. Shariff, E. D. Shirokoff, L. Stan, A. A. Stark, J. Sobrin, K. Story, A. Suzuki, Q. Y. Tang, R. B. Thakur, K. L. Thompson, C. E. Tucker, K. Vanderlinde, J. D. Vieira, G. Wang, N. Whitehorn, V. Yefremenko, and K. W. Yoon, Proc. SPIE 9914, 290-300 (2016).

${ }^{4}$ R. O’Brient, P. Ade, K. Arnold, J. Edwards, G. Engargiola, W. Holzapfel, A. T. Lee, X.-f. Meng, M. Myers, G. Rebeiz, P. Richards, H. Spieler, A. Suzuki, and H. T. Tran, AIP Conf. Proc. 1185, 502-505 (2009).

${ }^{5}$ F. Columbro, P. G. Madonia, L. Lamagna, E. S. Battistelli, A. Coppolecchia, P. de Bernardis, R. Gualtieri, S. Masi, A. Paiella, F. Piacentini, G. Presta, M. Biasotti, G. D’Alessandro, F. Gatti, L. Mele, and B. Siri, J. Low Temp. Phys. 199, 312-319 (2020).

${ }^{6}$ P. A. R. Ade, R. W. Aikin, M. Amiri, D. Barkats, S. J. Benton, C. A. Bischoff, J. J. Bock, J. A. Bonetti, J. A. Brevik, I. Buder, E. Bullock, G. Chattopadhyay, G. Davis, P. K. Day, C. D. Dowell, L. Duband, J. P. Filippini, S. Fliescher, S. R. Golwala, M. Halpern, M. Hasselfield, S. R. Hildebrandt, G. C. Hilton, V. Hristov, H. Hui, K. D. Irwin, W. C. Jones, K. S. Karkare, J. P. Kaufman, B. G. Keating, S. Kefeli, S. A. Kernasovskiy, J. M. Kovac, C. L. Kuo, H. G. LeDuc, E. M. Leitch, N. Llombart, M. Lueker, P. Mason, K. Megerian, L. Moncelsi, C. B. Netterfield, H. T. Nguyen, R. O’Brient, R. W. O. IV, A. Orlando, C. Pryke, A. S. Rahlin, C. D. Reintsema, S. Richter, M. C. Runyan, R. Schwarz, C. D. Sheehy, Z. K. Staniszewski, R. V. Sudiwala, G. P. Teply, J. E. Tolan, A. Trangsrud, R. S. Tucker, A. D. Turner, A. G. Vieregg, A. Weber, D. V. Wiebe, P. Wilson, C. L. Wong, K. W. Yoon, and J. Zmuidzinas, Astrophys. J. 812, 176 (2015).

${ }^{7}$ H. McCarrick, G. Jones, B. R. Johnson, M. H. Abitbol, P. A. R. Ade, S. Bryan, P. Day, T. Essinger-Hileman, D. Flanigan, H. G. Leduc, M. Limon, P. Mauskopf, A. Miller, and C. Tucker, Astron. Astrophys. 610, A45 (2018).

${ }^{8}$ S. Masi, P. de Bernardis, A. Paiella, F. Piacentini, L. Lamagna, A. Coppolecchia, P. A. R. Ade, E. S. Battistelli, M. G. Castellano, I. Colantoni, F. Columbro, G. D’Alessandro, M. D. Petris, S. Gordon, C. Magneville, P. Mauskopf, G. Pettinari, G. Pisano, G. Polenta, G. Presta, E. Tommasi, C. Tucker, V. Vdovin, A. Volpe, and D. Yvon, J. Cosmol. Astropart. Phys. 2019, 003.

${ }^{9}$ A. Paiella, A. Coppolecchia, L. Lamagna, P. A. R. Ade, E. S. Battistelli, M. G. Castellano, I. Colantoni, F. Columbro, G. D’Alessandro, P. de Bernardis, S. Gordon, S. Masi, P. Mauskopf, G. Pettinari, F. Piacentini, G. Pisano, G. Presta, and C. Tucker, J. Cosmol. Astropart. Phys. 2019(1), 039, arXiv:1810.00598 [astroph.IM].

${ }^{10}$ H. Sugai, P. A. R. Ade, Y. Akiba, D. Alonso, K. Arnold, J. Aumont, J. Austermann, C. Baccigalupi, A. J. Banday, R. Banerji, R. B. Barreiro, S. Basak, J. Beall, S. Beckman, M. Bersanelli, J. Borrill, F. Boulanger, M. L. Brown, M. Bucher, A. Buzzelli, E. Calabrese, F. J. Casas, A. Challinor, V. Chan, Y. Chinone, J.-F. Cliche, F. Columbro, A. Cukierman, D. Curtis, P. Danto, P. de Bernardis, T. de Haan, M. D. Petris, C. Dickinson, M. Dobbs, T. Dotani, L. Duband, A. Ducout, S. Duff, A. Duivenvoorden, J.-M. Duval, K. Ebisawa, T. Elleflot, H. Enokida, H. K. Eriksen, J. Errard, T. Essinger-Hileman, F. Finelli, R. Flauger, C. Franceschet, U. Fuskeland, K. Ganga, J.-R. Gao, R. Génova-Santos, T. Ghigna, A. Gomez, M. L. Gradziel, J. Grain, F. Grupp, A. Gruppuso, J. E. Gudmundsson, N. W. Halverson, P. Hargrave, T. Hasebe, M. Hasegawa, M. Hattori, M. Hazumi, S. Henrot-Versille, D. Herranz, C. Hill, G. Hilton, Y. Hirota, E. Hivon, R. Hlozek, D.-T. Hoang, J. Hubmayr, K. Ichiki, T. Iida, H. Imada, K. Ishimura, H. Ishino, G. C. Jaehnig, M. Jones, T. Kaga, S. Kashima, Y. Kataoka, N. Katayama, T. Kawasaki, R. Keskitalo, A. Kibayashi, T. Kikuchi, K. Kimura, T. Kisner, Y. Kobayashi, N. Kogiso, A. Kogut, K. Kohri, E. Komatsu, K. Komatsu, K. Konishi, N. Krachmalnicoff, C. L. Kuo, N. Kurinsky, A. Kushino, M. Kuwata-Gonokami, L. Lamagna, M. Lattanzi, A. T. Lee, E. Linder, B. Maffei, D. Maino, M. Maki, A. Mangilli, E. Martínez-González, S. Masi, R. Mathon, T. Matsumura, A. Mennella, M. Migliaccio, Y. Minami, K. Mistuda, D. Molinari, L. Montier, G. Morgante, B. Mot, Y. Murata, J. A. Murphy, M. Nagai, R. Nagata, S. Nakamura, T. Namikawa, P. Natoli, S. Nerval, T. Nishibori, H. Nishino, 
Y. Nomura, F. Noviello, C. O'Sullivan, H. Ochi, H. Ogawa, H. Ogawa, H. Ohsaki, I. Ohta, N. Okada, N. Okada, L. Pagano, A. Paiella, D. Paoletti, G. Patanchon, F. Piacentini, G. Pisano, G. Polenta, D. Poletti, T. Prouvé, G. Puglisi, D. Rambaud, C. Raum, S. Realini, M. Remazeilles, G. Roudil, J. A. Rubiño-Martín, M. Russell, H. Sakurai, Y. Sakurai, M. Sandri, G. Savini, D. Scott, Y. Sekimoto, B. D. Sherwin, K. Shinozaki, M. Shiraishi, P. Shirron, G. Signorelli, G. Smecher, P. Spizzi, S. L. Stever, R. Stompor, S. Sugiyama, A. Suzuki, J. Suzuki, E. Switzer, R. Takaku, H. Takakura, S. Takakura, Y. Takeda, A. Taylor, E. Taylor, Y. Terao, K. L. Thompson, B. Thorne, M. Tomasi, H. Tomida, N. Trappe, M. Tristram, M. Tsuji, M. Tsujimoto, C. Tucker, J. Ullom, S. Uozumi, S. Utsunomiya, J. V. Lanen, G. Vermeulen, P. Vielva, F. Villa, M. Vissers, N. Vittorio, F. Voisin, I. Walker, N. Watanabe, I. Wehus, J. Weller, B. Westbrook, B. Winter, E. Wollack, R. Yamamoto, N. Y. Yamasaki, M. Yanagisawa, T. Yoshida, J. Yumoto, M. Zannoni, and A. Zonca, "Updated design of the CMB polarization experiment satellite LiteBIRD," J. Low Temp. Phys. (published online 2020), arXiv:2001.01724 [astro-ph.IM]

${ }^{11}$ M. Salatino, P. de Bernardis, and S. Masi, Astron. Astrophys. 528, A138 (2011). ${ }^{12}$ F. Columbro, E. S. Battistelli, A. Coppolecchia, G. D’Alessandro, P. de Bernardis, L. Lamagna, S. Masi, L. Pagano, A. Paiella, F. Piacentini, and G. Presta, Astron. Nachr. 340, 83 (2019)

${ }^{13}$ L. Lamagna, G. Addamo, P. A. R. Ade, C. Baccigalupi, A. M. Baldini, P. M. Battaglia, E. Battistelli, A. Bau', M. Bersanelli, M. Biasotti, C. Boragno, A. Boscaleri, B. Caccianiga, S. Caprioli, F. Cavaliere, F. Cei, K. A. Cleary, F. Columbro, G. Coppi, A. Coppolecchia, D. Corsini, F. Cuttaia, G. D’Alessandro, P. de Bernardis, G. D. Gasperis, M. D. Petris, F. D. Torto, V. Fafone, Z. Farooqui, F. Farsian, F. Fontanelli, C. Franceschet, T. Gaier, F. Gatti, R. Genova-Santos, M. Gervasi, T. Ghigna, M. Grassi, D. Grosso, F. Incardona, M. Jones, P. Kangaslahti, N. Krachmalnicoff, R. Mainini, D. Maino, S. Mandelli, M. Maris, S. Masi, S. Matarrese, A. May, P. Mena, A. Mennella, R. Molina, D. Molinari, G. Morgante, F. Nati, P. Natoli, L. Pagano, A. Paiella, F. Paoness, A. Passerini, M. P. de Taoro, O. A. Peverini, F. Pezzotta, F. Piacentini, L. Piccirillo, G. Pisano, L. Polastri, G. Polenta, D. Poletti, G. Presta, S. Realini, N. Reyes, A. Rocchi, J. A. Rubino-Martin, M. Sandri, S. Sartor, A. Schillaci, G. Signorelli, M. Soria, F. Spinella, V. Tapia, A. Tartari, A. Taylor, L. Terenzi, M. Tomasi, E. Tommasi, C. Tucker, D. Vaccaro, D. M. Vigano', F. Villa, G. Virone, N. Vittorio, A. Volpe, B. Watkins, A. Zacchei, and M. Zannoni, "Progress report on the large scale polarization explore," J. Low Temp. Phys. (published online 2020).

${ }^{14}$ A. Mennella, P. Ade, G. Amico, D. Auguste, J. Aumont, S. Banfi, G. Barbaràn, P. Battaglia, E. Battistelli, A. Baù, B. Bélier, D. Bennett, L. Bergé, J. Bernard, M. Bersanelli, M. Sazy, N. Bleurvacq, J. Bonaparte, J. Bonis, E. Bunn, D. Burke, D. Buzi, A. Buzzelli, F. Cavaliere, P. Chanial, C. Chapron, R. Charlassier, F. Columbro, G. Coppi, A. Coppolecchia, R. D'Agostino, G. D'Alessandro, P. Bernardis, G. Gasperis, M. Leo, M. Petris, A. Donato, L. Dumoulin, A. Etchegoyen, A. Fasciszewski, C. Franceschet, M. Lerena, B. Garcia, X. Garrido, M. Gaspard, A. Gault, D. Gayer, M. Gervasi, M. Giard, Y. Héraud, M. Berisso, M. González, M. Gradziel, L. Grandsire, E. Guerard, J. Hamilton, D. Harari, V. Haynes, S. Versillé, D. Hoang, N. Holtzer, F. Incardona, E. Jules, J. Kaplan, A. Korotkov, C. Kristukat, L. Lamagna, S. Loucatos, T. Louis, A. Lowitz, V. Lukovic, R. Luterstein, B. Maffei, S. Marnieros, S. Masi, A. Mattei, A. May, M. McCulloch, M. Medina, L. Mele, S. Melhuish, L. Montier, L. Mousset, L. Mundo, J. Murphy, J. Murphy, C. O'Sullivan, E. Olivieri, A. Paiella, F. Pajot, A. Passerini, H. Pastoriza, A. Pelosi, C. Perbost, M. Perciballi, F. Pezzotta, F. Piacentini, M. Piat, L. Piccirillo, G. Pisano, G. Polenta, D. Prêle, R. Puddu, D. Rambaud, P. Ringegni, G. Romero, M. Salatino, A. Schillaci, C. Scóccola, S. Scully, S. Spinelli, G. Stankowiak, M. Stolpovskiy, F. Suarez, A. Tartari, J. Thermeau, P. Timbie, M. Tomasi, S. Torchinsky, M. Tristram, C. Tucker, G. Tucker, S. Vanneste, D. Viganò, N. Vittorio, F. Voisin, R. Watson, F. Wicek, M. Zannoni, and A. Zullo, Universe 5, 42 (2019).

${ }^{15}$ T. Essinger-Hileman, A. Kusaka, J. W. Appel, S. K. Choi, K. Crowley, S. P. Ho, N. Jarosik, L. A. Page, L. P. Parker, S. Raghunathan, S. M. Simon, S. T. Staggs, and K. Visnjic, Rev. Sci. Instrum. 87, 094503 (2016).

${ }^{16}$ B. R. Johnson, F. Columbro, D. Araujo, M. Limon, B. Smiley, G. Jones, B. Reichborn-Kjennerud, A. Miller, and S. Gupta, Rev. Sci. Instrum. 88, 105102 (2017).
${ }^{17}$ F. Columbro, P. de Bernardis, and S. Masi, Rev. Sci. Instrum. 89, 125004 (2018).

${ }^{18}$ Y. Sakurai, T. Matsumura, T. Iida, H. Kanai, N. Katayama, H. Imada, H. Ohsaki, Y. Terao, T. Shimomura, H. Sugai, H. Kataza, R. Yamamoto, and S. Utsunomiya, IEEE Trans. Appl. Supercond. 28, 1 (2018).

${ }^{19}$ See https://www.comsol.com/ for details on the COMSOL software.

${ }^{20} \mathrm{~F}$. Columbro, "The search for primordial B-modes in the polarization of the cosmic microwave background with LSPW/SWIPE and LiteBIRD," Ph.D. thesis, Sapienza, University of Rome, Italy, 2020.

${ }^{21}$ Y. Sekimoto, P. Ade, K. Arnold, J. Aumont, J. Austermann, C. Baccigalupi, A. Banday, R. Banerji, S. Basak, S. Beckman, M. Bersanelli, J. Borrill, F. Boulanger, M. L. Brown, M. Bucher, E. Calabrese, A. Challinor, Y. Chinone, F. Columbro, A. Cukierman, D. Curtis, P. de Bernardis, M. de Petris, M. Dobbs, T. Dotani, L. Duband, A. Ducout, K. Ebisawa, T. Elleflot, H. Eriksen, J. Errard, R. Flauger, C. Franceschet, U. Fuskeland, K. Ganga, J. Gao, T. Ghigna, J. Grain, A. Gruppuso, N. Halverson, P. Hargrave, T. Hasebe, M. Hasegawa, M. Hattori, M. Hazumi, S. Henrot-Versille, C. Hill, Y. Hirota, E. Hivon, D. T. Hoang, J. Hubmayr, K. Ichiki, H. Imada, H. Ishino, G. Jaehnig, H. Kanai, S. Kashima, Y. Kataoka, N. Katayama, T. Kawasaki, R. Keskitalo, A. Kibayashi, T. Kikuchi, K. Kimura, T. Kisner, Y. Kobayashi, N. Kogiso, K. Kohri, E. Komatsu, K. Komatsu, K. Konishi, N. Krachmalnicoff, C. L. Kuo, N. Kurinsky, A. Kushino, L. Lamagna, A. T. Lee, E. Linder, B. Maffei, M. Maki, A. Mangilli, E. Martinez-Gonzalez, S. Masi, T. Matsumura, A. Mennella, Y. Minami, K. Mistuda, D. Molinari, L. Montier, G. Morgante, B. Mot, Y. Murata, A. Murphy, M. Nagai, R. Nagata, S. Nakamura, T. Namikawa, P. Natoli, T. Nishibori, H. Nishino, F. Noviello, C. O'Sullivan, H. Ochi, H. Ogawa, H. Ogawa, H. Ohsaki, I. Ohta, N. Okada, G. Patanchon, F. Piacentini, G. Pisano, G. Polenta, D. Poletti, G. Puglisi, C. Raum, S. Realini, M. Remazeilles, H. Sakurai, Y. Sakurai, G. Savini, B. Sherwin, K. Shinozaki, M. Shiraishi, G. Signorelli, G. Smecher, R. Stompor, H. Sugai, S. Sugiyama, A. Suzuki, J. Suzuki, R. Takaku, H. Takakura, S. Takakura, E. Taylor, Y. Terao, K. L. Thompson, B. Thorne, M. Tomasi, H. Tomida, N. Trappe, M. Tristram, M. Tsuji, M. Tsujimoto, S. Uozumi, S. Utsunomiya, N. Vittorio, N. Watanabe, I. Wehus, B. Westbrook, B. Winter, R. Yamamoto, N. Y. Yamasaki, M. Yanagisawa, T. Yoshida, J. Yumoto, M. Zannoni, and A. Zonca, Proc. SPIE 10698, $106981 Y$ (2018).

${ }^{22}$ CERNOXTM is a trademark of Lake Shore Cryotronics, Inc.

${ }^{23} \mathrm{See}$ https://www.minicircuits.com/pdfs/MAR-8A+.pdf for the complete datasheet of the component.

${ }^{24}$ See https://www.analog.com/media/en/technical-documentation/data-sheets/ AD8361.pdf for the complete data-sheet of the component.

${ }^{25}$ L. Mele, P. Ade, J. G. Alberro, A. Almela, G. Amico, L. H. Arnaldi, D. Auguste, J. Aumont, S. Azzoni, S. Banfi, E. Battistelli, A. Bau', B. Belier, D. Bennett, L. Berge, J.-P. Bernard, M. Bersanelli, M.-A. Bigot-Sazy, N. Bleurvacq, J. Bonaparte, J. Bonis, A. Bottani, E. Bunn, D. Burke, D. Buzi, F. Cavaliere, P. Chanial, C. Chapron, R. Charlassier, F. Columbro, A. Coppolecchia, G. D’Alessandro, P. de Bernardis, G. D. Gasperis, M. D. Leo, M. D. Petris, S. Dheilly, L. Dumoulin, A. Etchegoyen, A. Fasciszewski, L. Ferreyro, D. Fracchia, C. Franceschet, M. G. Lerena, K. Ganga, B. Garcia, M. G. Redondo, M. Gaspard, D. Gayer, M. Gervasi, M. Giard, V. Gilles, Y. Giraud-Heraud, M. G. Berisso, M. Gonzalez, M. Gradziel, L. Grandsire, J.-C. Hamilton, D. Harari, S. Henrot-Versille, D. Hoang, F. Incardona, E. Jules, J. Kaplan, C. Kristukat, L. Lamagna, S. Loucatos, T. Louis, B. Maffei, S. Marnieros, W. Marty, S. Masi, A. Mattei, A. May, M. McCulloch, S. Melhuish, A. Mennella, L. Montier, L. Mousset, L. M. Mundo, J. A. Murphy, J. Murphy, F. Nati, E. Olivieri, C. Oriol, C. O'Sullivan, A. Paiella, F. Pajot, A. Passerini, H. Pastoriza, A. Pelosi, C. Perbost, M. Perciballi, F. Pezzotta, F. Piacentini, M. Piat, L. Piccirillo, G. Pisano, M. Platino, G. Polenta, D. Prele, R. Puddu, D. Rambaud, P. Ringegni, G. E. Romero, M. Salatino, J. Salum, A. Schillaci, C. Scoccola, S. Scully, S. Spinelli, G. Stankowiak, M. Stolpovskiy, A. Tartari, J.-P. Thermeau, P. Timbie, M. Tomasi, S. Torchinsky, M. Tristram, G. Tucker, C. Tucker, D. Vigano', N. Vittorio, F. Voisin, F. Wicek, M. Zannoni, and A. Zullo, "The QUBIC instrument for CMB polarization measurements," J. Phys.: Conf. Ser. (in press).

${ }^{26}$ G. D'Alessandro, L. Mele, F. Columbro, L. Pagano, F. Piacentini, P. de Bernardis, and S. Masi, Astron. Astrophys. 627, A160 (2019). 